\title{
IMPACTO DE UN MODELO TRIPLE HÉLICE EN EL CENTRO UNIVERSITARIO DEL SUR, UNIVERSIDAD DE GUADALAJARA
}

\section{Impact of a model triple bélix in a University of Guadalajara}

- Ricardo Xicoténcatl García Cauzor* Universidad de Guadalajara, México

Correo-e: rxgarcia@cusur.udg.mx

- Elba Rosa Gómez Barajas

Universidad de Guadalajara, México

Correo-e: elbagomez@valles.udg.mx
- José Manuel Ríos Ariza

Universidad de Guadalajara, México

Correo-e: jmrios@valles.udg.mx

\section{Resumen}

En el Centro Universitario del Sur (CUSur), de la Universidad de Guadalajara, ubicado en Ciudad Guzmán (Jalisco, México), se ha desarrollado, desde el ańo 2011, un proyecto de Triple Hélice en el que se han vinculado la Universidad, el gobierno local y el tejido empresarial de la Región Sur. La evaluación de los resultados y del proceso de la implementación de este proyecto es una de las claves para obtener información, que puede servir para la toma de decisiones y diseñar estrategias que permitan la optimización del funcionamiento de la triple hélice. La evaluación se ha centrado en diversos indicadores, como creación de empresas y puestos de trabajo, formación impartida, asesoramiento a empresarios, personas que han participado en las actividades, etc. Las herramientas utilizadas, los datos obtenidos y su interpretación exceden los límites de lo que es un artículo de investigación. Es por ello que nos vamos a centrar en uno de los aspectos menos estudiados en las experiencias de Triple Hélice: el impacto del modelo en el cambio organizacional, en nuestro caso, del Centro Universitario del Sur, con la creación de nuevas estructuras organizativas destinadas a la investigación.

Palabras clave: Triple Hélice; organización; universidad.

\begin{abstract}
At University of the South Central (CUSur) of the University of Guadalajara, located in Ciudad Guzmán (Jalisco, Mexico), has, since the year 2011, a project has developed of Triple Helix in which have been linked to the University, local government and the business sector of the Southern Region. Evaluation of the results and the process of the implementation of this project is one of the keys to obtain information, which can serve for decision-making and design strategies that allows the optimization of the performance of the triple helix. The evaluation has focused on various indicators as creation of enterprises and jobs, provided training, advice to entrepreneurs, people who have participated in the activities, etc. The tools used, the data obtained and their interpretation exceeds the limits of what is a research paper. Therefore, we're going to focus on one of the least studied aspects, on the experiences of Triple Helix, which is the impact of the model on organisational change, in our case, the University Center of South, with the creation of new organizational structures for research.
\end{abstract}

Keywords: Triple helix, organization, university.

*Autor de correspondencia.

ISSN (impreso): 2636-2139

ISSN (en línea): 2636-2147

Recibido: 6/06/2017

Sitio web: https://revistas.isfodosu.edu.do/recie

Aprobado: 22/08/2017

COMO CITAR:

García Cauzor, R. X., Gómez Barajas, E. R., \& Ríos Ariza, J. M. (2017). Impacto de un modelo triple hélice en el Centro Universitario del Sur, Universidad de Guadalajara. Revista Caribeña de Investigación Educativa (RECIE), 1(1), 7-17. https://doi.org/10.32541/recie.2017.v1i1.pp7-17 


\section{Revisión de la literatura}

\subsection{Concepto y surgimiento del modelo Triple Hélice}

A mediados de los años noventa se comienza a hablar, masivamente, de la necesidad de fomentar las interacciones dinámicas entre los factores Universidad-Empresa-Gobierno con el fin de acceder a la innovación y al desarrollo social.

El profesor Henry Etzkowitz estudió la importancia de unir estos tres factores con el propósito de fomentar el desarrollo de las regiones y ciudades. Este modelo es el camino ideal para que la universidad tradicional avance hacia el desarrollo de una universidad emprendedora, y aporte, de ese modo, mayores beneficios a la sociedad en sentido general (Etzkowitz, 1998).

$\mathrm{Al}$ respecto, Chang (2010, p. 7) afirma que "este modelo pretende que el accionar de la universidad sea un creador del conocimiento, que juega un papel primordial entre la relación empresa y gobierno (...) y cómo estos se desarrollan para crear innovación en las organizaciones como fuente de creación del conocimiento".

La importancia de la universidad como formadora integral de seres humanos y generadora de conocimiento, con el apoyo de las empresas y de los gobiernos con políticas encaminadas al emprendurismo, hace que este modelo siga vigente en estos tiempos difíciles en términos socioeconómicos, que exigen la cooperación de las tres hélices.

De acuerdo con González (2009, p. 17) "el papel de las universidades es estratégico en todo el proceso, pues se requiere que las universidades desempeñen nuevas funciones y que se generen instituciones diferenciadas. La tercera función de la universidad en las economías y sociedades del conocimiento es contribuir al desarrollo económico y social local mediante innovaciones basadas en el conocimiento".

Esto ha dado origen a la llamada "tercera revolución académica". Las denominadas universidades emprendedoras asumen la creación de empresas o de nidos empresariales en sus laboratorios, dando lugar a un nuevo tipo de personal docente y de investigador: el científico-empresario. Los estudiantes, en ellas, se ven como inventores potenciales y representan un flujo dinámico de capital humano en los grupos académicos de investigación. Este flujo constante asegura a las instituciones de educación superior (IES) como fuentes de innovación (Leydesdorff \& Etzkowitz, 2001, citados por González, 2009).

Hay dos posibles tipos de Triple Hélice. En el primero, la universidad, la industria y el gobierno están relacionados, pero cada uno conserva y desempeña su rol tradicional; en el segundo, hay una interpenetración en cada uno de estos componentes, y los actores antes mencionados asumen papeles que tradicionalmente correspondían a otros (por ejemplo, universidades que forman empresas), dando origen de esta forma a instituciones de carácter híbrido. El primer tipo es en esencia el mismo planteamiento teórico del triángulo de Sábato, expuesto treinta años antes. En el segundo, el aspecto novedoso lo marcan las zonas de intersección entre cada hélice, que es donde al final se generan las nuevas instituciones y se da el paso a la "universidad empresarial". Pero quizás la diferencia más evidente entre el planteamiento de Sábato y el de Etzkowitz se centre en que el primero es un planteamiento normativo, una directriz general para fomentar el desarrollo científico-tecnológico que, por tanto, no mostraba evidencia de que ello estuviera sucediendo en aquel momento; mientras 
que el segundo es un concepto descriptivo, que da cuenta de la dinámica real de la última década, evidenciada en los países desarrollados (Arocena \& Sutz, 2001).

La Triple Hélice no solo recalca el valor de las relaciones entre universidad, gobierno e industria, sino que también valora las transformaciones internas en cada una de esas esferas, y el modo en que comenzaron a gestionarse, hace algún tiempo, en los países desarrollados.

En América Latina, el proceso ha sido mucho más accidentado. La aplicación del modelo de Triple Hélice en nuestros países está íntimamente relacionado con el mejoramiento de las políticas nacionales en materia de ciencia y tecnología, la definición de los instrumentos necesarios para el desarrollo de estructuras acordes a las nuevas exigencias de la competitividad internacional y la definición de estímulos adecuados, en aras de favorecer la interrelación de los actores. Estas acciones deben contemplar la colocación de las instituciones de educación superior en un lugar especial, ya que como expresa Etzkowitz (1998, p. 53): "las nuevas exigencias y los cambios institucionales parecen reservarle a las universidades, en varias partes del mundo, un papel crecientemente importante".

Para integrar las tres esferas institucionales, los autores del concepto presentan las siguientes dimensiones:

1. Transformación interior de cada una de las hélices.

2. La influencia recíproca entre las mismas.

3. La creación de nuevos formatos de redes trilaterales y la sistematización de las interacciones entre ellas, con el objetivo de generar un proceso innovador de ideas y tecnologías para el desarrollo.

4. El efecto de estas transformaciones, estos intercambios y estas nuevas redes generarán innovaciones que beneficiarán de modo creciente a la sociedad.

Cada una de las hélices puede asumir el papel de otra ya sea creando incubadoras de empresas, organizando la innovación regional (universidad), asumiendo una dimensión académica, compartiendo conocimientos (empresa), realizando investigación y promoviendo las mutuas interrelaciones (gobierno). La Triple Hélice se puede caracterizar como un modelo de eslabones múltiples, en constante evolución, en el que, en cada una de las fases del proceso de innovación, se aprovecha al máximo el conocimiento que genera cada una de las hélices. Estas tres esferas, que antes trabajaban de manera independiente, tienden a trabajar en conjunto.

Los actores que intervienen en él son los investigadores académicos, convertidos en gestores y aplicadores de sus propias tecnologías, los empresarios que trabajan en un laboratorio universitario o una oficina de transferencia tecnológica, los investigadores públicos, los investigadores académicos y los investigadores industriales, que dirigen agencias regionales responsables de la transferencia tecnológica.

Esta convergencia de las esferas institucionales académicas, industriales y gubernamentales, en la que cada una asume el papel de las otras, ha sido interpretada de diferentes formas en distintas partes del mundo. En países donde la interfaz marcha bien, sea que ocurra de abajo arriba, a través de las interacciones de individuos y organizaciones de diferentes esferas institucionales, o de arriba abajo, estimulada por decisiones políticas, se le considera un fenómeno empírico. Estados Unidos ha sido visto como un ejemplo de la primera modalidad, y Europa un ejemplo de la última modalidad de aplicación (Viale \& Campbell'Orto, 2000).

Por otra parte, la Triple Hélice ha mostrado su potencialidad para contribuir a la solución de los problemas de tipo social y medioambiental. Por un lado, exportando el modelo hacia la creación de 
otras triples hélices que atiendan a problemas sociales acuciantes en distintas partes del planeta. Por otro, poniendo a trabajar a las entidades Triple Hélice en soluciones científicas y tecnológicas (lo que incluye ciencias y tecnologías sociales como soluciones organizativas o entornos normativos) a problemas demandados por una organización, la sociedad civil o las administraciones públicas en sus múltiples escalas (de lo local a lo internacional) (Etzkowitz \& Zhou, 2006).

Vista a la luz de este modo de afrontar el asunto, la innovación conlleva dos aspectos: uno, el de la aplicación e implementación del modelo de manera creativa; y el otro, de gran trascendencia para la Universidad, el impacto que produce sobre la práctica educativa y los estilos de enseñanza-aprendizaje que tienden a promover, como ha acontecido en el Centro Universitario del Sur.

Según Ríos y Gómez (2011, p. 204) la innovación es “un cambio que se produce deliberadamente, con una finalidad y en el que se diseñan estrategias para conseguir su implementación. Desde esta conceptualización, evidentemente, toda innovación supone un cambio, pero no todo cambio es una innovación", (...) "ésta surge de una idea propia que se inicia una vez que se rompe con la resistencia propia al cambio. Posteriormente, habrá que socializarla de forma tal que se convenza a un grupo de las ventajas que tiene desaprender lo aprendido y dejar atrás lo obsoleto. Esas ideas deben transformarse en un algún producto, proceso o servicio que sea susceptible de ser usado, compartido, vendido y/o aplicado" (p. 206).

Siguiendo a Domingo (2013), la innovación es constituyente cuando en ella se da la reflexión, la comprensión local, el compromiso profesional y la acción colectiva de la comunidad para ofrecer respuestas consistentes a la mejora del aprendizaje de todos. Es decir, antes de pensar siquiera en la implementación de cualquier proyecto tecnológico, de innovación que logre un impacto en la sociedad, en las empresas o en las políticas públicas, se debe pensar en el contexto en el que se pretende implementar tal proyecto, sus características socioculturales y la respuesta que puede dar al proyecto la gente que interactúa en este espacio.

\subsection{Evaluación del impacto del modelo Triple Hélice}

La construcción de sistemas de indicadores, para evaluar, se convierte en un reto dada la complejidad de los diferentes sistemas de innovación. Freeman y Soete (2009) señalan que los indicadores relacionados con la ciencia y la tecnología están siendo construidos y utilizados, pero que se puede caer en el abuso, ya que la facilidad actual parar acceder a recursos informáticos en estadística ha hecho común la utilización de indicadores. Sin embargo, la posibilidad de poder emplearlos de manera comparativa fracasa por depender de contextos, fundamentos y métodos diferentes.

El Manual de Oslo (OCDE, 2005) está entre los principales en extenderse y aplicarse. La cienciometría, las encuestas, entrevistas, la observación, los casos de estudio, econometría, benchmarking, análisis de clúster y crecimientos de la $\mathrm{I}+\mathrm{D}$, etc., se han venido fusionando para organizar la evaluación en forma sistémica, en total correspondencia con las características y demandas de los sistemas de ciencia e innovación. El énfasis en la evaluación de los impactos socioeconómicos de la investigación está relacionado en particular con expectativas crecientes que conciernen a los impactos económicos de financiamiento de la investigación, y por la responsabilidad y deseos de demostrar tales impactos cuantitativamente. Esto ha conducido a esfuerzos en el desarrollo de resultados cuantitativos y medidas de impacto (Luukkonen, 2002). 
Para Molas-Gallart, Tang y Morrow (2000), el impacto puede ser, según su naturaleza, directo o indirecto. El indirecto tiene influencias a corto o largo plazos. A este tipo de impacto Estébanez (2002) le denomina impacto potencial. Ambos autores, aun con formas distintas de denominarlos, coinciden en que las contribuciones indirectas inciden de manera significativa en la toma de decisiones y la adopción de políticas.

El impacto social de la ciencia y la tecnología implica identificar cambios, transformaciones. Según Quevedo, Chía y Rodríguez (2002), los efectos de la ciencia y la innovación tecnológica pueden concebirse como el cambio o conjunto de cambios duraderos que se producen en la sociedad, la economía, la ciencia, la tecnología y el medio ambiente, que mejoran sus indicadores como resultado de la ejecución de acciones de $\mathrm{I}+\mathrm{D}+\mathrm{i}$ e introducen valor agregado a los productos, servicios, procesos y tecnologías.

Sin embargo, existen sistemas en los que es posible distinguir dos tipos de indicadores: los que valoran la actividad y los que se refieren a su incidencia en la universidad y sus procesos internos (D' Este, Castro \& Molas-Gallart, 2009).

Los indicadores de actividad miden el trabajo y el esfuerzo de la universidad con las comunidades no académicas; y los de impacto, los resultados de estas interacciones en términos sociales y del desarrollo económico.

Los indicadores utilizados por D'Este, Castro y Molas-Gallart (2009) están agrupados en las siguientes categorías: emprendimiento (creación de empresas); asesoramiento y consultoría; movilidad de personal; prácticas en empresas; cursos y actividades de formación; alineamiento curricular; participación en programas o redes sociales, y difusión no académica. Muchos de estos indicadores y categorías han sido utilizados en la evaluación del modelo Triple Hélice implementado en la Región Sur de Jalisco.

La aplicación de un solo tipo de indicadores haría parcial e insuficiente la evaluación, pues sesgaría los resultados al no considerar los demás elementos y factores de un proceso tan complejo, como la relación interinstitucional de colaboración a que nos estamos refiriendo. No obstante, como hemos indicado anteriormente, aunque hemos evaluado las distintas categorías, en este artículo ponemos especial énfasis en una modalidad que no suele ser tomada en cuenta a la hora de medir el impacto de los proyectos de Triple Hélice, y que vamos a detallar más adelante.

De hecho, no existe un modelo de evaluación del impacto de las relaciones de vinculación de la universidad que pueda considerarse único y aplicable a todos los casos. Cada institución educativa, a partir de su contexto y sus alianzas estratégicas, deberá desarrollar un sistema de medición particular que le permita, desde sus peculiaridades, analizar el impacto de su actividad de colaboración extrainstitucional, en razón de las metas específicas establecidas por ella y sus colaboradores, lo que redundará en aportes conceptuales y metodológicos al conocimiento de este tipo de vínculos.

\section{Contexto de la universidad de Guadalajara}

La Universidad de Guadalajara (México) vive un momento de cambio que ha quedado plasmado en su Plan de Desarrollo 2014-2030. En él se reconoce la necesidad de reorientar las funciones sustantivas de la docencia, la investigación, la extensión y la vinculación para lograr un desarrollo armónico e integral, que posibilite a la institución enfrentar los retos y desafíos que tiene la universidad pública en el siglo xxI. 
Desde 1994, está conformada por 15 centros universitarios, de los cuales seis están ubicados en la zona metropolitana de Guadalajara y los restantes, nueve, en las regiones del Estado de Jalisco (México). Los centros universitarios regionales, desde su creación, fueron concebidos con la intención de que quedaran ubicados en una determinada región, integrada por varios municipios, de forma tal que en la misión de cada centro se expresa la necesidad de dar cobertura y apoyo a los municipios de su área de influencia. Esta particular situación conlleva la necesidad de que los centros universitarios regionales sean pensados como motores del desarrollo educativo, social, cultural y económico de las regiones en las que tienen su sede.

El Centro Universitario del Sur (CUSur) es un centro regional ubicado en la Región Sur del Estado de Jalisco, su sede está en Zapotlán el Grande (Ciudad Guzmán) y su área de influencia abarca 28 municipios de las regiones Sur y Sureste del estado.

Su relación con esta importante cantidad de municipios ha sido un factor decisivo para generar acciones de vinculación con los diferentes sectores sociales, productivos y gubernamentales, lo que permite el desarrollo de actividades que contribuyen al beneficio y mejoramiento de la calidad de vida de los habitantes de esta región.

A principios de 2009 la Unión Europea impulsó proyectos de vinculación, con algunos países de Latinoamérica, con la finalidad de fortalecer las diferentes áreas de desarrollo económico del continente. Impulsados por el Ayuntamiento de Irún y la Junta de Castilla y León, Espańa, así como Bidasoa Activa/Bidasoa Bizirik, y URB-AL, se pone en marcha el proyecto denominado "UNE: Estrategias de desarrollo local incluyentes y participativas", proyecto mediante el cual se vincularon con México, Chile, Honduras y Perú.

En 2010 se recibió en CUSur la invitación, del Ayuntamiento de Zapotlán el Grande, para participar de un proyecto auspiciado por la UNE, siguiendo el modelo de la "Triple Hélice", como una herramienta técnica que viabiliza la colaboración y el trabajo participativo del gobierno municipal, los empresarios y la universidad (Centro Universitario del Sur e Instituto Tecnológico de Ciudad Guzmán).

El proyecto que desarrollarían en Zapotlán se titulaba "Diseño de la metodología de colaboración entre la Universidad-Empresa-Gobierno local para impulsar la investigación aplicada al desarrollo tecnológico en el campo empresarial en la realidad del municipio de Zapotlán el Grande, en el marco del proyecto UNE”.

Para CUSur resultó importante participar de un esquema que pretendía fortalecer la vinculación universitaria con el tejido empresarial y con el gobierno local. Con ello se pretendió dar vitalidad a la investigación científica y tecnológica, para que se lograra una correcta transferencia hacia los actores empresariales y la sociedad, bajo el liderazgo del gobierno municipal.

El proyecto estuvo orientado a generar una metodología en la que los tres actores principales (Universidad-Gobierno-Empresa) se vincularan adecuadamente y conformaran un equipo de colaboración autogestivo, multidisciplinario y autodirigido, para que los resultados de estas entidades impactaran directamente en el desarrollo sustentable de la comunidad.

Para la elaboración de la propuesta metodológica de vinculación desarrollada en el 2011, se realizaron las siguientes actividades:

Fase inicial:

1. Se elaboró un estudio de las metodologías de la Triple Hélice que se han aplicado nacional e internacionalmente. 
2. Se realizó un diagnóstico sobre las condiciones que existían, en materia de vinculación, entre las instituciones de educación superior, el sector empresarial y el gobierno de Zapotlán el Grande.

Fase de implementación:

Se generó, a través de investigadores de las dos instituciones educativas, una metodología básica para la operación del modelo de la Triple Hélice. Esta ha servido, desde el año 2012, como marco para las diferentes acciones de trabajo. Además, se trabajó en la estructuración de los proyectos correspondientes a tres áreas fundamentales para consolidar la vinculación:

1. La creación de una incubadora de base tecnológica interinstitucional.

2. La creación de una Red de Centros de Negocios con el propósito de aglutinar las unidades operativas ubicadas en las instituciones de educación superior (IES) de la región y de articular los procesos administrativos y económicos orientados a consolidar a las empresas locales.

3. La creación y/o incorporación de laboratorios de investigación aplicada para fortalecer la vinculación del personal académico y de los estudiantes de las IES con el sector empresarial, e impulsar la competitividad local.

El modelo contempló una temporalidad de 20 años, con actividades a desarrollar a corto, mediano y largo plazos. En el tiempo de ejecución que lleva se puede asegurar que ha impactado de manera notoria los sectores relacionados como la economía, la salud, la cultura y la educación, entre otros.

Por lo general, los estudios relacionados con la medición del impacto de modelos de vinculación Universidad-Gobierno-Empresa privilegian los acercamientos a los resultados producidos en materia económica. De igual forma, existen numerosos estudios que analizan y evalúan el rol de cada uno de los actores de esta vinculación, con énfasis en los que revisan el papel de las empresas y el gobierno para favorecer, impulsar e incluso frenar las relaciones de vinculación que se desarrollan bajo modelos de este tipo.

En esta investigación reviste central importancia observar la interface científica y tecnológica de la Universidad, para constatar si la academia sufrió algún proceso de replanteamiento teórico, metodológico o bien de organización estructural y funcional producto de esta relación estratégica de colaboración interinstitucional.

\section{Método}

El objetivo de la investigación fue evaluar el impacto del modelo de vinculación de Triple Hélice, en el Centro Universitario del Sur, para confirmarlo como una innovación educativa con impacto en las funciones de investigación, extensión y vinculación que realiza esta institución educativa.

En este artículo sólo vamos a centrarnos en la evaluación del impacto del modelo de la Triple Hélice en la estructura de organización académica, vinculada a la investigación, del Centro Universitario del Sur, concretamente en lo que vamos a denominar "plataforma científica y tecnológica del CUSur".

La investigación es descriptiva. El trabajo de investigación se realizó en base a los principios epistemológicos de la investigación holística, que integra diferentes perspectivas e instrumentos. En la metodología holística, como seńala Hurtado de Barrera (2000), los modelos epistémicos de investigación pueden tener una función complementaria y no necesariamente son contradictorios.

Se emplearon técnicas e instrumentos que posibilitaron la recogida de información en los centros de investigación responsables de la producción y transferencia de tecnología. 
Las herramientas e instrumentos utilizados han sido: (a) cuestionario; (b) Entrevistas con los responsables de los centros de investigación que se desarrollaron de manera estructurada, tomando como base el cuestionario; (c) Análisis de documentos de los centros de investigación, grupos de investigación y redes de vinculación; (d) Entrevistas con los cuerpos académicos y redes de vinculación.

El cuestionario estaba estructurado con preguntas mixtas, en las que se consideran respuestas cerradas y abiertas. El cuestionario se construyó con dos bloques de reactivos: el primero, relativo a los datos generales (con respuestas cerradas) constituido por catorce reactivos sobre la información básica que permite identificar, el centro de investigación, su origen, capacidades productivas, recursos humanos, trayectoria y articulación académica; el segundo abarca once reactivos relacionados con la vinculación (respuestas abiertas), para identificar las actividades de interacción y de trabajo colaborativo del centro de investigaciones con otras entidades académicas y no académicas, y la participación de la unidad académica en estrategias de vinculación interinstitucional.

A partir de la información obtenida, mediante el análisis de documentos, de las entrevistas y cuestionarios, se procedió a la elaboración de fichas básicas de cada centro de investigación y a la elaboración del diagnóstico de las fortalezas y debilidades (FODA), propuesto en la metodología, debido a la necesidad de reconocer si cada instancia académica (centro de investigación) tiene una imagen clara de su situación, mediante la evaluación de sus potencialidades y sus debilidades; así como para determinar si han sido tomadas en cuenta las áreas de oportunidad y las posibles amenazas que puede enfrentar. Todo lo anterior se hizo con el propósito de obtener una radiografía de la estructura académica, entendida como la plataforma que sustenta la definición de las áreas estratégicas de desarrollo, que desde cada una de ellas se plantean para el futuro a corto, mediano y largo plazos.

Las dimensiones que se contemplaron en la plataforma científica y tecnológica de CUSur son:

- Centros de investigación. Unidad departamental que realiza investigación. Debe contar, por lo menos, con dos académicos de carrera, con la categoría de titular o el grado de doctor, con el propósito de que desarrollen dos líneas fundamentales de investigación.

- Cuerpos académicos (CA). Figura académica reconocida por el Consejo Nacional de Ciencia y Tecnología de México (CONACYT), que consiste en la agrupación de investigadores que tienen como fin la producción colectiva de conocimiento y su aplicación. Son grupos de profesores de tiempo completo que comparten una o varias líneas de generación y aplicación innovadora del conocimiento (LGAC) (investigación o estudio) en temas disciplinares o multidisciplinares, así como un conjunto de objetivos y metas académicas comunes. Dado que en muchos países se les denomina Grupos de Investigación, en el resto del documento vamos a referirnos a ellos con esta denominación.

- Redes de vinculación. En esta investigación, se entiende por ello las vías de comunicación que establecen las estructuras académicas del CUSur, tanto al interior del Centro Universitario como con su exterior.

\section{Resultados y conclusiones}

Debido al pequeño número de entrevistas, fichas básicas elaboradas y diagnósticos FODA no es

pertinente hacer un análisis de datos de tipo estadístico, sino una interpretación en base a categorías 
que exponemos en este apartado de resultados y conclusiones. Igualmente, dada la singularidad de la evaluación realizada y de las dimensiones que hemos utilizado, y puesto que no hemos usado los indicadores usuales (mencionados en la parte teórica), en el análisis del impacto del modelo de Triple Hélice, no procede la comparación de los datos obtenidos en CUSur con la implementación de este modelo en otras universidades.

El análisis de la ficha básica y los diagnósticos FODA, realizados a cada uno de los centros de investigación, permitió conocer que las seis nuevas unidades académicas (Centros de Investigación) cuentan con una estructura sólida para el desarrollo de líneas y proyectos de investigación, todas claramente definidas y con objetivos pertinentes. Asimismo, cuentan con excelentes plantillas de investigadores, muchos de ellos con posgrados y una amplia producción científica. También, se verificaron sus vínculos con otras instancias académicas y sus estrategias de colaboración con instituciones no académicas (gobierno y empresas).

La creación de seis centros de investigación durante el lapso comprendido entre el 2012 y el 2015 es una evidencia del aumento de la capacidad y las actividades de producción de conocimiento y transferencia de tecnología en el CUSur, acaecidos durante la implementación del modelo de Triple Hélice.

De igual forma, es destacable el incremento de los grupos de investigadores en el Centro Universitario del Sur, ya que desde su creación en 1994 y hasta 2008 sólo contaba con tres grupos de investigación. En cuatro años se han creado y desarrollado nueve grupos de investigación, debido a la necesidad de combinar la creación de grupos y centros de investigación. Esta relación presenta una gran alineación pues los nueve cuerpos académicos creados, en el periodo de ejecución del programa tienen correspondencia y adscripción a un centro de investigación.

La creación de una red de vinculaciones académicas coloca a cada uno de los centros como una unidad de tracción y conexión con otras instancias académicas, como los laboratorios de docencia que dan apoyo principalmente a las licenciaturas; los grupos de investigación; los posgrados y las relaciones con instancias no académicas externas al centro universitario. Las redes de vinculación, de cada centro de investigación, tienen conexión con el resto de centros, con lo que se constató también una nueva estructura de comunicación científica en el CUSur.

En el análisis realizado se pudo constatar el fortalecimiento de los estudios de posgrado durante este periodo. El Centro Universitario sólo contaba con la Maestría de Administración de Negocios (2007), en tanto que en 2011 creó la Maestría en Ciencias de la Salud Pública, la maestría y el doctorado en Comportamiento Alimentario y Nutrición (2011 y 2013, respectivamente), la maestría y el doctorado en Psicología y Calidad de Vida (2015), aparte de varios posgrados más en procesos de aprobación. Es importante destacar que la investigación permitió constatar que las propuestas antes mencionadas surgen articuladas a los centros de investigación descritos y analizados.

Los cinco espacios universitarios en cuestión, al ser analizados en su estructura, organización y producción, aparecen sólidamente ligados a actividades de extensión, y vinculados con sectores externos a la Universidad, académicos y no académicos. También se pudo comprobar que tres de las cinco instancias académicas descritas están consideradas como parte de la agenda de actividades que se diseñó conforme al modelo de vinculación desarrollado para Zapotlán el Grande.

La investigación realizada aporta material fundamental para constatar que la estructura académica del Centro Universitario del Sur ha sufrido una importante transformación de la plataforma científica y tecnológica. La investigación permitió observar que en el CUSur se reconoció que la 
existencia de un solo centro de investigación (CICAN, 2009) no era suficiente para desarrollar conocimiento, ni integraba los diversos campos de conocimiento que el centro universitario cimentaba desde sus programas de licenciatura y posgrado, lo que impulsó a la identificación de nuevos nichos de desarrollo de conocimiento científico, ligados a necesidades y problemas concretos de la región, sin perder de vista su impacto a nivel nacional e internacional.

La puesta en marcha de proyectos como este y otros que avancen en la misma línea, para poder desarrollarse de forma pertinente e incidir en el contexto, no solo requieren de la participación de la Universidad como actora, sino que ésta transforme sus estructuras organizativas. La vinculación de los profesores con la empresa y el gobierno local, y con las comunidades, reorienta y alumbra necesidades y evidencia objetos de estudio que pueden abrir nuevas líneas de investigación y dar paso a la creación de estructuras organizativas que permitan la puesta en marcha y el funcionamiento de la labor investigadora.

La Universidad está inserta en un mundo cambiante, en el que es previsible el crecimiento de la economía social, fruto de la colaboración entre ciudadanos, la disminución del empleo y la deslocalización (Rifkin, 2015). El modelo de Triple Hélice (enriquecido con la participación y el protagonismo de las comunidades y la sociedad civil, cuarta hélice) puede convertirse en un instrumento de cohesión social y ayudar a dar respuestas a los cambios que vayan surgiendo.

La Universidad debe reforzar su papel local (desarrollo endógeno) sin perder de vista su visión y su misión globales, y mantener su protagonismo como generadora de conocimiento. Si este componente llegara a fallar, la investigación quedaría solo en poder de las grandes compañías, dando lugar a unos oligopolios, a los que solo entrarían las grandes corporaciones

La Universidad es imprescindible para las grandes empresas multinacionales, holding y grandes corporaciones, pero en el otro lado de la balanza están las pequeñas y medianas empresas, con frecuencia más conectadas a las necesidades locales y a las comunidades, por cuanto no pueden ser desatendidas. Proyectos como el que exponemos favorecen el desarrollo en una localidad y una región.

No obstante, el protagonismo de las Universidades podría quedar opacado si el profesorado no se implica, y si las estructuras orgánicas y la organización de las Universidades no son flexibles. Esta flexibilidad ha incidido en CUSur en:

- La incorporación de investigadores a la Triple Hélice, ayudando a la consecución de sus objetivos.

- La creación de nuevas líneas de investigación y estructuras organizativas en CUSur.

\section{Referencias}

Arocena, A, \& J. Sutz. (2001). La Universidad Latinoamericana del futuro. Tendencias, Escenarios, Alternativas. México: UDUAL.

Chang, H. G. (2010). El modelo de la triple hélice como un medio para la vinculación entre la universidad y la empresa. Revista Nacional de Administración, 1(1), 85-94. Recuperado de https://bit.ly/2kNfVCO

D’ Este A., Castro E. \& Molas-Gallart J. (2009). Manual de Indicadores de Vinculación de la universidad con el entorno socioeconómico. Buenos Aires: OCTS. Recuperado de https://bit. ly/2Lk99Q8 
Domingo, J. (2013). Un marco crítico de apoyo para ubicar y redireccionar experiencias innovadoras en educación: comprensión y transformación. Tendencias pedagógicas, 21, 9-28. Recuperado de https://bit.ly/2Lm9YYP

Estebánez, M. E. (2002). Impacto social de la ciencia y la tecnología: estrategia para su análisis, incluido en El Estado de la Ciencia. Principales Indicadores de Ciencia y Tecnología Iberoamericanos/ Interamericanos. Recuperado de https://bit.ly/2LUS7ZK

Etzkowitz, H. (1998). The norms of entrepreneurial science: cognitive effects of the new university-industry linkages. Research policy, 27(8), 823-833. Recuperado de https://bit.ly/2HiDhbY

Etzkowitz, H. \& Zhou, C. (2006). Triple Helix twins: innovation and sustainability. Science and Public Policy, 33 (1), 77-83. Recuperado de https://bit.ly/2JccAaT

Freeman, C. \& Soete, L. (2009). Developing science, technology and innovation indicators: What we can learn from the past. Research Policy, 38(4), 583-589. Recuperado de https://bit.ly/2LUfL95

González de la Fe, T. (2009). El modelo de triple hélice de relaciones universidad, industria y gobierno: un análisis crítico. ARBOR Ciencia, Pensamiento y Cultura, 738, 739-755 Recuperado de https://bit.ly/2MfXQND

Hurtado de Barrera, J. (2000). Metodología de investigación holística. Caracas: Editorial SYPAL.

Luukkonen, T. (2002). Research evaluation in Europe: state of the art. Research Evaluation, 11(2), $81-84$.

Molas-Gallart, J., Tang, P., \& Morrow, S. (2000). Assessing the non-academic impact of grant-funded socioeconomic research: results from a pilot study. Research Evaluation, 9(3), 171-182.

OCDE (2005). The Measurement of Scientific and Technological Activities. Proposed Guidelines for Collecting and Interpreting Technological Innovation Data. París: OECD. Recuperado de https://bit.ly/2EfVs4D

Rifkim, J. (2015). La sociedad del costo marginal cero. El Internet de las cosas, el procomún colaborativo y el eclipse del capitalismo. México: Paidós.

Ríos, J. M. \& Gómez, E. R. (2011). Innovación en un mundo globalizado: dos casos. En Hernández, R.; Padilla, S. y Arancibia, M. (coords.), Educación Superior, Innovación e Internacionalización. Ensayos, proyectos y experiencias (pp. 203-240). Guadalajara: Universidad de Guadalajara y Universidad Austral de Chile.

Viale, R. \& Campodall'Orto, S. (April, 2000). Neocorporations or Evolutionary Triple Helix?

Suggestions Coming from European Regions, in the Third Triple Helix Conference, Rio de Janeiro. 